\title{
Rainfall variability and adverse birth outcomes in Amazonia
}

Erick A. Chacón-Montalván ${ }^{1}$, Benjamin M. Taylor ${ }^{2}$, Marcelo G. Cunha ${ }^{3}$, Gemma Davies ${ }^{4}$, Jesem D.Y. Orellana ${ }^{5}$, Luke Parry ${ }^{4,6 *}$

10

${ }^{1}$ Department of Mathematics and Statistics, Lancaster University, UK

${ }^{2}$ Blackpool Teaching Hospitals NHS Foundation, UK

${ }^{3}$ Escola Nacional de Saúde Pública, Fundação Oswaldo Cruz, Rio de Janeiro, Brazil

${ }^{4}$ Lancaster Environment Centre, Lancaster University, UK

${ }^{5}$ Instituto Leônidas e Maria Deane, Fundação Oswaldo Cruz, Manaus, Brazil

${ }^{6}$ Núcleo de Altos Estudos Amazônicos, Universidade Federal do Pará, Belém, Brazil * corresponding author 
Amazonian populations are increasingly exposed to climatic shocks yet knowledge of related health impacts is limited. Understanding how health risks are co-produced by local climatic variability, place and social inequities is vital for improving decision-making, particularly in decentralized contexts. We assess the impacts of rainfall variability and multi-scale vulnerabilities on birth-weight, which has life-long health consequences. We focus on highly river-dependent areas in Amazonia, using urban and rural birth registrations during 20062017. We find a strong but spatially-differentiated relationship between local rainfall and subsequent river-level anomalies. Using Bayesian models we disentangle the impacts of rainfall shocks of different magnitudes, municipal characteristics, social inequities and seasonality. Prenatal exposure to extremely intense rainfall is associated with preterm birth, restricted intra-uterine growth and lower mean birth-weight $(\leq-183 g)$. Adverse birth outcomes also follow non-extreme intense rainfall (40\% higher odds of low birth-weight), drier than seasonal averages (-39g mean birth-weight) and conception in the rising-water season (-13g mean birth-weight). Babies experience penalties totalling 646g when born to adolescent, Amerindian, unmarried mothers that received no formal education, antenatal or obstetric healthcare. Rainfall variability confers inter-generational disadvantage, especially for socially-marginalized Amazonians in forgotten places. Structural changes are required to reduce inequities, foster citizen empowerment, and improve the social accountability of public institutions. 
Amazonian societies were exposed to major floods in 2009, 2012, 2013 and 2014 and severe droughts in 2005, 2010 and $2015^{1-3}$, causing widespread disruption to flows of essential goods and public services ${ }^{4,5}$. These increasingly frequent and severe events are caused by extremely intense or deficient rainfall associated with La Niña and El Niño events, and changes in water transport to Amazonia linked to anomalies in sea surface temperatures in the tropical Atlantic ${ }^{6}$. Vulnerability to climatic shocks is particularly high among Amazonia's marginalized river-dependent populations, including remote 'jungle towns' unconnected to road networks ${ }^{7}$. Yet, despite intensive biophysical research into how extreme events in Amazonia influence, and respond to, forest degradation and planetary change (e.g. $\left.{ }^{2}\right)$, the health impacts of climatic variability in the region are underresearched $^{8}$. These knowledge gaps arises from intersecting forms of marginalization through which research communities and policy-makers neglect certain kinds of places and peoples ${ }^{9}$.

Increasing climatic variability in Amazonia is concerning, in part, because prenatal exposure to climatic shocks can harm birth outcomes ${ }^{10-12}$ and early life climatic shocks may hinder childhood development ${ }^{13-15}$. Consequences of low birth-weight $(\mathrm{LBW} ;<2500 \mathrm{~g})$, for example, include subsequent disadvantages in educational attainment, health and income in adulthood, and mortalityrisks $^{16-19}$. Work highlights three main mechanisms linking climatic variability with birth outcomes (including fetal growth and pregnancy duration). First, climatic shocks affect crop yields and quality, which can cause food insecurity and malnutrition by constraining food access among rural agricultural populations or urban consumers ${ }^{20,21}$. Second, these shocks can alter the survival and reproduction of vectors and pathogens, compromising maternal health through increased incidence of water-borne ${ }^{11}$ or vector-borne disease ${ }^{22}$. Third, shock-related stress, anxiety or mental health disorders during pregnancy can cause preterm birth $^{23}$ and impair child development ${ }^{24}$. It is challenging, however, to disentangle the inter-connected effects of these pathways ${ }^{25}$. For example, evidence linking disasters with stress and birth outcomes in the Global South is inconclusive and 
confounded by impacts on maternal health and nutrition ${ }^{26,27}$. Furthermore, although prenatal stress may cause preterm birth ${ }^{23}$, it can also restrict fetal growth ${ }^{26}$. And, whilst maternal nutrition is important for fetal growth ${ }^{28}$, it also influences risks of preterm birth ${ }^{29,30}$.

Rainfall variability in Amazonia affects local environments, economic activity and social systems in complex ways ${ }^{5}$, with myriad consequences for physical and psychosocial health. For instance, intense rainfall can cause extreme flooding, which exacerbates water insecurity ${ }^{31}$, increases risks of malaria ${ }^{32}$ and diarrhoeal disease ${ }^{33}$ and jeopardizes floodplain agriculture ${ }^{34}$. The health consequences of these, latter, short-term agricultural impacts are not well understood - research in the Global South has mainly examined changes in average climatic conditions ${ }^{35}$. The effects of the malnutrition, disease, and stress pathways are inter-linked and exacerbated because extreme climatic events also damage social systems, displace households, and disrupt mobilities, employment and access to public services ${ }^{36}$, including healthcare ${ }^{35}$. Even lower-magnitude variability in rainfall and river-levels in Amazonia can affect access to natural resources ${ }^{37}$ and riverine transport and trade along sub-tributaries, with implications for livelihoods and food security ${ }^{5}$. In Amazonia, as elsewhere, vulnerability is likely to be greatest for those living in multidimensional poverty ${ }^{38}$ although the relative importance of inter-linked causal mechanisms may be place-specific.

Local-scale variation in climate-health risks is poorly understood, which is problematic because climatic stressors affect health through interactions with contextual and structural factors (e.g. political, economic, cultural, environmental $)^{39}$ and socio-economic inequities. This may explain spatial variation in the vulnerability of social and food systems to climatic shocks ${ }^{7}$. Place also matters due to decentralization of local governance of healthcare systems, emergency infrastructure and humanitarian services, and longer-term investment in adaptation in many countries ${ }^{20}$. 
Furthermore, biophysical analysis of Amazonian floods and droughts tends to assess river-levels in the central mainstem (e.g. ${ }^{1}$ ) even though underlying episodes of anomalous rainfall are spatiallydistinct and affect sub-basins differently ${ }^{40}$. Considering locality is also important because of hydrological seasonality, which shapes Amazonia's social-ecological systems ${ }^{5,41}$. The timing reflects flood pulses of different rivers and varies spatially due to North-South hemisphere opposition in rainfall regimes ${ }^{42}$.

This paper questions how birth-weight in Amazonia is associated with maternal exposure to rainfall variability and social disadvantage. Given the interconnected vulnerabilities ${ }^{5}$ and limited related research in this system ${ }^{8}$, we examine associations between rainfall variability and birth-weight in order to stimulate future work, instead of hypothesis-testing a specific causal pathway(s). Our focus is on urban and rural newborns in 43 highly river-dependent municipalities in Amazonas State, Brazil (E.D. Fig. 1), vulnerable to climatic change ${ }^{7}$ and home to 1.278 million people. Our approach enables us to evaluate how an individual newborn's prenatal exposure to locally-occurring climatic variability and multi-scale inequities may reduce birth-weight via growth restriction or preterm birth (PTB) (E.D. Fig. 2; Methods). We separately evaluate how positive and negative rainfall shocks of varying magnitude affect birth outcomes, instead of using cumulative rainfall during pregnancy ${ }^{11}$ or during birth year ${ }^{43}$. We do not directly translate local rainfall anomalies as river-level anomalies, because the latter are influenced by rainfall variability elsewhere in the basin, and other hydrological processes (e.g. soil moisture, vegetation type).

We first calculate seasonal anomalies in satellite-based measures of local rainfall and river-level observations. Then, we separately calculate prenatal exposure to rainfall variability for each livebirth recorded between 2006-2017 based on maternal municipality of residence, birth-date and estimated conception date. Rainfall variability is evaluated weekly for each municipality using our 
model-based version of the Standardized Precipitation Index ${ }^{44}$. We assess cumulative anomalies in intense or deficient rainfall by identifying: (i) weekly deviation from seasonal averages; and over the preceding 8-weeks (ii) non-extreme events ( $>1 \mathrm{SD},<-1 \mathrm{SD}$ above the mean); and (iii) extreme events $(\geq 1.96 \mathrm{SD}, \leq-1.96)$ in each municipality (Fig. 1). For each newborn we calculate weeks of prenatal exposure (including the pre-pregnancy trimester) to each kind of rainfall variability (E.D.

Fig. 3). We define hydrological seasons separately for each municipality using historical river-level information (E.D. Fig. 4). Finally, we use hierarchical Bayesian modelling to disentangle the effects of the three levels of rainfall anomalies, hydrological seasonality, municipal characteristics (remoteness from other urban centres, and sanitation coverage) and maternal disadvantage on birth outcomes. We model: overall effects on birth-weight and LBW odds; effects on intra-uterine growth (birth-weight and LBW, controlling for gestational age); effects on PTB odds. Official birth certificates provided birth-weight, categorized gestational age, sex, newborn ethnicity and birthsetting, maternal characteristics and antenatal care received. Our individual-level analysis links the location and scale parameters of birth outcomes with linear predictors that depend on covariates, non-linear functions of covariates, and municipality and temporal effects. Due to the complexity of our models, the linear predictors account for the effects of municipality and maternal covariates and rainfall variability in an additive way.

\section{$<$ FIGURE $1>$}

\section{Relationship between rainfall and river-level anomalies}

We find a correlation of 0.29 between rainfall anomalies and river-level anomalies, with a mean lag time of 4 weeks from the former to the latter (E.D. Fig. 5). Rainfall anomalies are closely related $(0.49<\mathrm{r}<0.59)$ with river-level anomalies in the Negro sub-basin (E.D. Fig. 6a), which is mostly 
Purús Rivers (E.D. Fig. 6c) whose sub-basins are mostly within Amazonas and Acre (Fig. 2).

Correlations are generally lower in municipalities along the main river-stem because the discharge is strongly influenced by rainfall upstream, in Peru, Ecuador and Colombia. Lag periods are shortest in the upper Negro River (1-to-3 weeks), Atalaia do Norte (1 week), and the upper Juruá and Purus Rivers (1-to-3 weeks), and longest (several months) in the east, around the main-stem (e.g. Parintins [15 weeks]; E.D. Fig. 5c).

\section{$<$ FIGURE 2>}

160 Overall, our results show that intense and deficient rainfall shocks are broadly indicative of subsequent river-level fluctuations in the upper sections of tributaries, where hydrological regimes appear more dependent on local rainfall. Around the main-stem, river-level anomalies are caused less by local rainfall and more by rainfall in areas upstream, hence long lag times (e.g. 3-4 months ${ }^{6}$ ). In the Bolivian Amazon local flood events occur due to flood waves from upriver and abundant local rainfall ${ }^{45}$. They also find local rainfall is a strong predictor of flooded area. In Amazonas, many rural river-dwellers live along third-order sub-tributaries strongly influenced by local rainfall ${ }^{46}$, and many urban centres are located near the mouths of these sub-tributaries. On the main-stem, an extreme hydrological event may not be detected using local rainfall, exemplified by our failure to identify flooding in Fonte Boa in $2015^{47}$ although we did capture the extremely intense rainfall further upriver, where river flooding also occurred and municipalities declared states of emergency ${ }^{48}$.

Some shocks were geographically-restricted (e.g. extremely intense rainfall events in 2015 in the far west) whereas others affected virtually all municipalities (e.g. extremely deficient rainfall events in 2015 (Fig. $3 ;{ }^{2}$ ). Many municipalities experienced multiple extreme rainfall events during the study 
period (S.M. Fig. 1), sometimes in consecutive years (Fig. 1). The distinct spatial signatures of the rainfall shocks demonstrate the limitations of determining basin-wide extreme events using only main-stem river-levels (e.g. $\left.{ }^{1}\right)$.

\section{Gestational age and birth-weight}

GA strongly influences birth-weight, hence controlling for GA enabled us to assess the independent effects of rainfall variability on growth (size-for-gestational-age). Compared to term pregnancies ( $\geq 37$ weeks), PTB reduced birth-weight by between -247g (CI:-254 to -241g) (32-to-36 weeks), 853g (CI:-881 to -827) (28-to-31 weeks) and -1774g (CI:-1820 to -1728) (22-to-27 weeks). LBW odds ratios for these GAs (compared to full-term) increased by 4.78 (CI: 4.59 to 4.97), 22.84 (CI: 20.76 to 25.12 ), and 47.57 (CI: 39.91 to 55.97$)$.

\section{Impacts of rainfall variability}

High exposure to extremely intense rainfall was associated with a $183 \mathrm{~g}$ reduction (CI:-319 to -57) in mean birth-weight (Fig. 4a), linked to increased risk of PTB (OR 222 ,CI: 16 to 894)(Fig. 4e). Growth was unaffected (Fig. 4b,d). Moderate exposure to extremely intense rainfall was associated with $60 \mathrm{~g}$ lower mean birth-weight (CI:-110 to -11$)$, or $-36 \mathrm{~g}$ (CI:-66 to -10) controlling for gestational age (GA)(Fig. 4b), indicating growth restriction. LBW odds were 94\% higher (CI:1.296 to 2.829; Fig. 4c) following this exposure but not significantly different when controlling for GA. Moderate exposure also reduced PTB odds substantially (CI:0.001 to 0.011). Evidence for extremely deficient rainfall events was weaker although we found significantly increased PTB odds (4.377, CI:1.01 to 11.872) and high probability (0.918) of increased LBW odds. Exposure to both rainfall extremes was associated with 3638 (CI:224 to 15561) higher PTB odds and no significant changes in birth-weight, although increasing LBW odds was more likely (0.866). Some double 
exposure was associated with reduced PTB risks, higher mean birth-weight and lower LBW risks (E.D. Fig. 7m,a,g, respectively), indicating complex changes in pregnancy outcomes.

Non-extreme intense rainfall events were associated with $40 \%$ increased LBW odds (CI:1.01 to 1.91), related to elevated PTB risks (1.79 OR, CI:1.12 to 2.67)(Fig. 4). Deficient episodes were associated with 55\% lower PTB odds (CI: 0.18 to 0.93 ) but no change in birth-weight. Very high exposure to dry episodes was associated with reduced PTB odds (E.D. Fig. 7n). Particular combinations of intense and deficient rainfall were associated with 15 -to-30g lower mean birthweight and 10-to-50\% higher LBW odds (E.D. Fig. 7b,h). These effects were related to PTB and growth restriction (E.D. Fig. 7e,k). Unusually low exposure to intense and deficient rainfall was associated with 10-to-30g lower mean birth-weight and higher LBW odds, mainly due to growthrestriction (E.D. Fig. 7b,e,h).

Drier-than-normal conditions were associated with 39g lower mean birth-weight (CI:-1 to -89), increased likelihood of LBW (0.919 exceedance probability), and 12.79 higher PTB odds (CI:7.03 to 21.49)(Fig. 4). Conversely, wetter-than-normal conditions were only associated with a high probability (0.92) of increased LBW odds. Combinations of unusually wet and dry periods were associated with 25\% higher LBW odds (CI:1.01 to 1.59), linked to higher PTB risks (OR 2.78, CI:1.66 to 4.45)(E.D. Fig. 7i,o). Nonetheless, double exposure may also restrict growth given an exceedance probability 0.894 of higher LBW odds, controlling for GA. Closeness to long-term seasonality was associated with $\sim 10$-to-20g increased size-for-gestational-age (E.D. Fig. 7). Exposure to major deviations from long-term seasonal rainfall trends significantly increased PTB odds (E.D. Fig. 7o). 
$<$ FIGURE $4>$

Extremely intense rainfall in Amazonia is associated with severely reduced mean birth-weight due to PTB and restricted growth. Risks are greatest following months of exceptional rainfall, which often causes river flooding. Urban and rural communities in our study area are highly riverdependent and have experienced a five-fold increase in flood frequency in recent decades ${ }^{1}$. However, we show that intense but non-extreme rainfall is also harmful due to PTB and, when combined with intense dry periods, restricts growth. Overall, our results support the suggestion that nutrition in 'wetter' regions is more vulnerable to excessive rainfall than to drought ${ }^{20}$. At low magnitude, however, variability in drier conditions appears relatively more important. These findings build on evidence from the Global South. In the Brazilian semi-arid, lower rainfall during pregnancy is correlated with higher infant mortality, lower birth-weight and premature birth ${ }^{11}$. However, their estimated effects were relatively minor; one SD increase in rainfall (28\%) was associated with $1.6 \mathrm{~g}$ higher birth-weight. In rural Mexico, more extreme wet or dry seasons limit children's growth ${ }^{49}$ and in rural Vietnam, low rainfall during pregnancy and infancy limits heightfor-age $e^{50}$. In Indonesia, there are health and socio-economic benefits of higher rainfall during the first year of life, whereas no apparent effects of rainfall variability in utero $^{43}$. Exposure to flooding during first year of life in India is associated with higher rates of stunting and underweight in children ${ }^{14}$.

\section{Municipal-scale determinants}

Mean birth-weight was 44g lower (CI:-81 to -6) in remote municipalities (0.8/1.0 remoteness score) with low sanitation (15\% of households)(E.D. Fig. 8). Additionally, higher LBW odds was more 
likely (0.93) and odds were $22 \%$ higher (CI:1.01 to 1.42) when controlling for GA, indicating impaired growth. These remote places are $\geq 959 \mathrm{~km}$ river distance from nearest state capital, which can takes weeks by boat ${ }^{7}$. Sanitation is less common in more remote municipalities (correlation $\mathrm{r}=$ 0.53), therefore their joint effects are more meaningful. Nonetheless, low sanitation was associated with growth restriction (-32g [CI:-2 to -61]; lower size-for-gestational age) and 25\% lower odds of PTB (CI:0.57 to 0.98), potentially indicating suppressed fertility or more miscarriages. Remoteness was associated with high probability (0.94) of increasing LBW odds. Beyond sanitation, remoteness underlies higher population sensitivity and lower adaptive capacity for coping with climatic shocks linked to higher imported food prices, reduced institutional presence and effectiveness, and governance failures in education and healthcare ${ }^{7}$. Summarizing, municipal characteristics have sizeable marginal effects on birth-weight, controlling for maternal covariates.

Birth outcomes still differ markedly by municipality (S.M. Fig. 2), after controlling for remoteness, sanitation, random effects and maternal covariates. For instance, mean birth-weight was $213 \mathrm{~g}$ higher and LBW odds 39\% lower in Carauari than in Envira, largely unchanged when controlling

265 for GA (E.D. Fig. 9). However, we cannot disentangle the role of contextual factors affecting maternal nutrition (e.g. dependence on floodplain agriculture, fisheries resources), from sociopolitical determinants of fetal health and survival chances (e.g. access to quality healthcare, disease burden, discrimination) or potential biases in measurement or reporting of birth-weight.

\section{Transmission of maternal disadvantage}

Social inequities within highly river-dependent Amazonian municipalities are strongly associated with lower birth-weight, confirming the inter-generational transmission of disadvantage ${ }^{16}$. For adolescent mothers aged 15, LBW odds are 2.09 times (CI:1.99 to 2.20) the odds of mothers 30 years old, and mean birth-weight is $248 \mathrm{~g}$ lower (CI:-242 to $-254 \mathrm{~g}$ )(Fig. 5a-c). These effects are 
robust to GA, although young mothers also have $66 \%$ higher odds of PTB (CI:1.58 to 1.74).

Mothers with no formal education have smaller babies than those with $1-3$ years $(28 \%$ higher LBW odds [CI:1.19 to 1.38 ] and 63g lower mean [CI:-55 to -72]) or 4-7 years of education (33\% higher LBW odds [CI:1.25 to 1.43 ] or $84 \mathrm{~g}$ lower mean [CI:-76 to -93$])$. These effects are robust to GA although no education was associated with 9\% increased PTB odds (CI for 1-to-3 year comparison; 1.02 to 1.16$)$. Being unmarried carried a penalty of $17 \%$ higher odds of LBW (CI:1.11 to 1.24$)$ and $32 \mathrm{~g}$ lower mean birth-weight (CI:-26 to -37). These effects are robust to GA and PTB odds were not significantly different. Indigenous Amerindian ethnicity was associated with 7\% higher LBW odds (CI:1.02 to 1.12) and 58g lower mean birth-weight (CI:-52 to -63). Indigenous newborns had 30\% higher odds of PTB (CI:1.24 to 1.36), although size-for-gestational-age was still 50g lower (CI:-44 to -55). Summarizing, social inequities are strongly associated with restricted growth and more modest increases in PTB risks.

Receiving no antenatal care incurred 1.88 higher LBW odds (CI:2.70 to 3.08) and 151g lower mean birth-weight ([CI:-143 to $-159 \mathrm{~g}]$, compared to $\geq 7$ consultations. These effects were robust to GA although PTB odds were 1.70 higher (CI:1.58 to 1.82) with no consulations. Antenatal care may help avoid PTB but the linkage between no antenatal care and severe growth restriction suggests these mothers face other, unmeasured disadvantages (e.g. extreme poverty, remote rural residence). Mothers receiving no antenatal care give birth to lighter babies when exposed to extremely deficient rainfall events (S.M. Fig. 3). Home-birth babies were 74g lighter (CI:-69 to -79) than those born in hospital, with no significant difference in LBW odds. PTB odds for home-birth were $23 \%$ higher (CI:1.19 to 1.28) but size-for-gestational-age was still 63g lower (CI:-58 to -68).

Cumulative disadvantage for babies of adolescent, Amerindian, unmarried mothers with no formal education who had received neither antenatal or obstetric care equated to LBW odds 10.2 higher 
300 (CI:8.98 to 11.48 ) and 646g lower mean birth-weight (CI:632 to 660) than newborns of relatively advantaged mothers. Controlling for GA, the combined penalties are LBW OR 7.61 (CI:6.76 to 8.56) and 609g lower mean birth-weight (CI:-594 to -623), highlighting severely restricted growth. Nonetheless, their PTB risks are also 4.99 times greater (CI:4.44 to 5.55). These social inequities are prevalent within our study population (SI Table 1). For instance, 31.3\% of babies had mothers aged 19 years or less. Thirty-one percent of mothers had at least 7 antenatal consultations, with 4to-6 the most common (39.8\%) followed by 1-to-3 (21.9\%), or none $(6.4 \%)$. Summarizing, the susceptibility to harm from rainfall shocks in Amazonia is shaped by steep socio-economic gradients and their effects on disadvantaged newborns.

\section{Hydrological seasonality}

Hydrological seasons correlated with birth-weight (Fig. 5d-f), including 6g (-0.2 to -12) lower mean birth-weight following conception in the low-water season, compared to the high-water season. Controlling for GA, conception during the rising-water enchente season incurred $13 \mathrm{~g}$ reduced growth (CI: -7 to -19$)$ and 9\% higher LBW odds (CI: 1.033 to 1.143). Enchente-related growth

315 restriction may reflect malnutrition in trimesters one and two during the high-water season when there is poor fishing and food insecurity ${ }^{37}$. Early enchente conception was associated with $29 \%$ lower PTB OR (CI: 0.673 to 0.746$)$. Better growth following high-season conception may reflect favourable food access during the low-water season ${ }^{37}$ (second trimester). However, seasonality in birth outcomes could also reflect variation in other unmeasured factors such as healthcare access, 320 disease burden, etc. Nonetheless, these findings confirm seasonality in birth-weight ${ }^{51}$ and the vital role of rivers in this region.

$<$ FIGURE 5> 


\section{Discussion}

Our paper shows that maternal exposure to extreme rainfall shocks is associated with lower birthweight due to preterm birth and restricted growth. Consequently, rainfall shocks confer intergenerational disadvantage for river-dependent populations living in neglected areas of Amazonia. These marginalized populations experience injustice because, despite contributing little to climate change, they are responsible for safeguarding most remaining forest and highly susceptible to climatic shocks. Studies elsewhere found birth outcomes compromised by variation in rainfall ${ }^{11}$ and temperature ${ }^{10,51}$ yet our paper provides a significant advance by accounting for individual variance in magnitude of exposure to short-duration rainfall shocks. We show extreme rainfall shocks in Amazonas correlate with local river flooding or drought, although this linkage is stronger in upstream second-order tributaries and third-order tributaries and weaker along the main-stem.

\section{Extreme events exceed coping capacities}

Our findings suggest that Amazonian coping strategies (e.g. growing fast-growing crops to cope with flooding ${ }^{52}$ ) are, alone, insufficient for adapting to increased rainfall variability and subsequent 340 hydrological anomalies ${ }^{5}$. Moreover, under-resourced municipal governments appear unable to ensure the human right to health when faced with climatic extremes. Decentralization was intended to strengthen public services by improving resource allocation, accountability and local mobilization $^{53}$, but ineffective municipal structures hinder health and development in the South ${ }^{54}$. Non-extreme rainfall variability and subtler deviations in seasonal dry periods was also linked to lower birth-weight, however, pointing to the unfolding of 'slow', out-of-sight emergencies ${ }^{55}$.

\section{Reduce inequities and improve accountability}

Policy interventions to reduce vulnerability to climatic shocks include increasing the coverage of antenatal and obstetric care, investing in transportation and infrastructure to enable rural teenagers 
to complete high-school, and expanding the coverage of household sanitation. Current deficiencies

in these services reflect deep social inequities and the long-term political neglect of provincial Amazonia ${ }^{9}$, and uneven development in Brazil. Alleviating the double burden of climate change and health inequities requires structural changes to reduce inequities in power, money and resources and improve people's daily living conditions ${ }^{56}$. Amazonian populations also need improved early warning systems for floods ${ }^{57}$ which should be transparent and provide locally-relevant, timely and actionable information that is accessible to citizens, considering disparities in access to technology. These systems can promote greater social accountability of public institutions and empowerment of citizens, akin to a reaching out for greater power ${ }^{9}$.

\section{Future research}

Designing effective public health interventions will require understanding specific causal pathways linking birth outcomes with meteorological and hydrological shocks. For instance, research in highincome countries identifies trimester-specific effects of maternal stress ${ }^{26,58}$ and future Amazonian research could investigate how the timing of flood exposure determines the relative importance of the stress pathway on child development. The timing of exposure may also help understand a nutritional pathway, given observational studies showing that fetal growth and PTB risks are most associated with maternal nutrition in pre-pregnancy and the first trimester ${ }^{28}$. We may under-estimate the true effects of rainfall variability and seasonality on birth-weight given that rainfall variability, flooding and seasonal hardship can depress fertility and cause miscarriages ${ }^{11,59}$. This potential selection bias might also explain why some combinations of rainfall shocks appeared protective against PTB. In addition, healthcare resources are limited in our study context and most mothers received insufficient antenatal care, likely affecting the reliability of GA assessment ${ }^{60}$. Some studies report a tendency to under-estimate GA in growth-restricted newborns ${ }^{61}$, hence reduced growth may be more important than our results suggest. Finally, studying climate impacts on larger numbers of 
Amazonian municipalities may enable researchers to identify any independent effects of temperature extremes on birth-outcomes, as found elsewhere e.g. 11,49.

This work shows how birth-weight can be severely reduced by maternal exposure to extreme climatic shocks, creating life-long disadvantage when combined with place-based and social marginalization. Extreme rainfall events are responsible for subsequent river floods and droughts and pose greater risks for vulnerable mothers, especially those living in remote river-dependent places. Most climate-health research in Amazonia has focussed on the more accessible Estuary region (e.g. ${ }^{62}$ ) and work in Amazonas is scarce ${ }^{32}$. Beyond Amazonia, we provide novel insights by focussing on a highly river-dependent rural and urban population with multifaceted vulnerability to climate change. Moreover, we evidence why it is important to consider spatial variation in hazard exposure and vulnerability, and relative maternal disadvantage. Finally, this study shows how rainfall variability can severely affect birth-weight, demonstrating the urgent need to dedicate greater scientific resources and policy attention to enable Amazonians to better cope with climatic change.

\section{Data Availability}

The data that supports the findings of this study are publicly available as follows:

- Birth data from the Brazilian Information System for Live Births (Sistema de Informação Sobre

Nascidos Vivos, SINASC),

http://www2.datasus.gov.br/DATASUS/index.php?area=0901\&item $=1 \&$ acao $=28 \& p a d=31655$

- Municipality-level covariates from the Brazilian Institute of Geography and Statistics (Instituto Brasileiro de Geografia e Estatistica, IBGE), ftp://ftp.ibge.gov.br/Censos/Censo_Demografico_2010/Sinopse/Agregados_por_Setores_Censitario 400 s/ 
- Precipitation data from the Integrated Multi-satellitE Retrievals for GPM (IMERG)

https://pmm.nasa.gov/data-access/downloads/gpm

- River-level data from Brazil's National Water Agency (Agência Nacional de Águas, ANA), https://www.snirh.gov.br/hidroweb/publico/apresentacao.jsf

\section{Code Availability}

All analyses were performed using the open-source platform $\mathrm{R}$, version 4.0.2. We used the $m b s i$ package (https://github.com/ErickChacon/mbsi) to compute the model-based standardised precipitation index and the bamlss package (https://cran.rproject.org/web/packages/bamlss/index.html) to perform inference on the BAMLSS models. All the scripts for modelling can be found at https://gitlab.com/ErickChacon/birthweight, and visualize at https://erickchacon.gitlab.io/birthweight/. Additional code for data gathering, cleaning and processing; or processed data can be provided upon request.

\section{References (including for the Methods)}

1.Barichivich, J. et al. Recent intensification of Amazon flooding extremes driven by strengthened Walker circul 2.Yang, J. et al. Amazon drought and forest response: Largely reduced forest photosynthesis but slightly increas 3.Espinoza, J. C. et al. The extreme 2014 flood in south-western Amazon basin: the role of tropical-subtropical s 4.Marengo, J. A. et al. Recent extremes of drought and flooding in Amazonia: vulnerabilities and human adaptat 5.Pinho, P., Marengo, J. \& Smith, M. Complex socio-ecological dynamics driven by extreme events in the Amaz 6.Marengo, J. A. \& Espinoza, J. C. Extreme seasonal droughts and floods in Amazonia: causes, trends and impa 7.Parry, L. et al. Social vulnerability to climatic shocks is shaped by urban accessibility. Annals of the American 8.Brondízio, E. S., de Lima, A. C., Schramski, S. \& Adams, C. Social and health dimensions of climate change 1 9.Parry, L. et al. The (in)visible health risks of climate change. Social Science \& Medicine 241, 112448 (2019). 10.Andalón, M., Azevedo, J. P., Rodríguez-Castelán, C., Sanfelice, V. \& Valderrama-González, D. Weather shoc 11.Rocha, R. \& Soares, R. R. Water scarcity and birth outcomes in the Brazilian semiarid. Journal of Developme 12.Hilmert, C. J., Kvasnicka-Gates, L., Teoh, A. N., Bresin, K. \& Fiebiger, S. Major flood related strains and pre 13.Alderman, H. Safety Nets Can Help Address the Risks to Nutrition from Increasing Climate Variability. $J N u$ 14.Rodriguez-Llanes, J. M., Ranjan-Dash, S., Degomme, O., Mukhopadhyay, A. \& Guha-Sapir, D. Child malnu 15.Woldehanna, T. Do pre-natal and post-natal economic shocks have a long-lasting effect on the height of 5-ye 16.Aizer, A. \& Currie, J. The intergenerational transmission of inequality: Maternal disadvantage and health at $b$ 17.Oreopoulos, P., Stabile, M., Walld, R. \& Roos, L. L. Short-, Medium-, and Long-Term Consequences of Poor 18.Kramer, M. S. The Epidemiology of Low Birthweight. Maternal and Child Nutrition: The First 1,000 Days 7 
19.Wang, S.-F. et al. Birth weight and risk of coronary heart disease in adults: a meta-analysis of prospective col 20.Cooper, M., Brown, M. E., Azzarri, C. \& Meinzen-Dick, R. Hunger, nutrition, and precipitation: evidence fro 21.Grace, K., Brown, M. \& McNally, A. Examining the link between food prices and food insecurity: A multi-le 22.Beeson, J. G., Scoullar, M. J. L. \& Boeuf, P. Combating low birth weight due to malaria infection in pregnan 23.Kramer, M. S. et al. Stress pathways to spontaneous preterm birth: the role of stressors, psychological distres 24.Graignic-Philippe, R., Dayan, J., Chokron, S., Jacquet, A.-Y. \& Tordjman, S. Effects of prenatal stress on feta 25.Lindsay, K. L., Buss, C., Wadhwa, P. D. \& Entringer, S. The Interplay between Maternal Nutrition and Stress 26.Buffa, G. et al. Prenatal stress and child development: A scoping review of research in low- and middle-incor 27.Sanguanklin, N. et al. Effects of the 2011 Flood in Thailand on Birth Outcomes and Perceived Social Suppor 28.Ramakrishnan, U., Grant, F., Goldenberg, T., Zongrone, A. \& Martorell, R. Effect of Women's Nutrition befo 29.Kibret, K. T., Chojenta, C., Gresham, E., Tegegne, T. K. \& Loxton, D. Maternal dietary patterns and risk of a 506-520 (2019).

30.Bloomfield, F. H. How Is Maternal Nutrition Related to Preterm Birth? Annual Review of Nutrition 31, 235-2 31.Rosinger, A. Y. Household water insecurity after a historic flood: Diarrhea and dehydration in the Bolivian A 32.Wolfarth-Couto, B. et al. Variabilidade dos casos de malária e sua relação com a precipitação e nível d'água c 33.Fonseca, P., Hacon, S. de S. \& Reis, V. O uso de dados de satelite para estudar a relação entre chuva e doença 34.Chibnik, M. Risky rivers: the economics and politics of floodplain farming in Amazonia. (University of Arizo 35.Phalkey, R. K., Aranda-Jan, C., Marx, S., Höfle, B. \& Sauerborn, R. Systematic review of current efforts to q 36.Haines, A., Kovats, R. S., Campbell-Lendrum, D. \& Corvalan, C. Climate change and human health: Impacts 37.Tregidgo, D. J., Barlow, J., Pompeu, P. S. \& Parry, L. Tough fishing and severe seasonal food insecurity in Ar 38.Hallegatte, S. \& Rozenberg, J. Climate change through a poverty lens. Nature Climate Change 7, 250 (2017). 39.Berry, H. L., Waite, T. D., Dear, K. B., Capon, A. G. \& Murray, V. The case for systems thinking about clima 40.Espinoza, J. C., Ronchail, J., Marengo, J. A. \& Segura, H. Contrasting North-South changes in Amazon wet41.Langill, J. C. \& Abizaid, C. What is a bad flood? Local perspectives of extreme floods in the Peruvian Amazc 42.Espinoza Villar, J. C. et al. Spatio-temporal rainfall variability in the Amazon basin countries (Brazil, Peru, B 43.Maccini, S. \& Yang, D. Under the weather: Health, schooling, and economic consequences of early-life rainf 44.Chacon-Montalvan, E., Luke, P., Davies, G. \& Taylor, B. A model-based general alternative to the standardis 45.Ovando, A. et al. Extreme flood events in the Bolivian Amazon wetlands. Journal of Hydrology: Regional St 46.Parry, L., Peres, C. A., Day, B. \& Amaral, S. Rural-urban migration brings conservation threats and opportun 47.Brilhante, Nelson. Cheia histórica: 42 municípios estão em situação de emergência no Amazonas | Amazônia 48. Toledano, Diego \& Lifstich, Andrezza. Seca do Rio Negro ganha força e muda paisagens em Manaus. Globo 49.Skoufias, E. \& Vinha, K. Climate variability and child height in rural Mexico. Economics \& Human Biology 50.Thai, T. Q. \& Falaris, E. M. Child schooling, child health, and rainfall shocks: Evidence from rural Vietnam. 51.Strand, L. B., Barnett, A. G. \& Tong, S. The influence of season and ambient temperature on birth outcomes: 52.Sherman, M., Ford, J., Llanos-Cuentas, A., Valdivia, M. J. \& Bussalleu, A. Vulnerability and adaptive capacit 53.Guanais, F. C. \& Macinko, J. The Health Effects Of Decentralizing Primary Care In Brazil. Health Affairs 28 54.Harpham, T. Urban health in developing countries: what do we know and where do we go? Health \& place 1 55.Anderson, B., Grove, K., Rickards, L. \& Kearnes, M. Slow emergencies: Temporality and the racialized biop 56. Friel, S. Climate Change and the People's Health. (Oxford University Press, 2019).

57.Dottori, F. et al. Increased human and economic losses from river flooding with anthropogenic warming. Nat 58.Hoffman, M. C., Mazzoni, S. E., Wagner, B. D., Laudenslager, M. L. \& Ross, R. G. Measures of Maternal St 59.Mallett, L. H. \& Etzel, R. A. Flooding: what is the impact on pregnancy and child health? Disasters 42, 43260.Lee, A. C. et al. Validity of Newborn Clinical Assessment to Determine Gestational Age in Bangladesh. Pedi 61.Lee, A. C. et al. Diagnostic Accuracy of Neonatal Assessment for Gestational Age Determination: A Systema 62.de Lima, A. C. et al. Climate hazards in small and medium cities in the Amazon Delta and Estuary: challenge 63.Ibisch, P. L. et al. A global map of roadless areas and their conservation status. Science 354, 1423-1427 (2010 64.Gadelha, A. N. et al. Grid box-level evaluation of IMERG over Brazil at various space and time scales. Atmo. 65.Oliveira, R., Maggioni, V., Vila, D. \& Morales, C. Characteristics and Diurnal Cycle of GPM Rainfall Estima 66.Rozante, J. R., Vila, D. A., Barboza Chiquetto, J., Fernandes, A. D. A. \& Souza Alvim, D. Evaluation of TRN 67.Zubieta Barragán, R., Getirana, A., Espinoza, J. C., Lavado-Casimiro, W. \& Aragon, L. Hydrological modelin 
68.Molina-Carpio, J. et al. Hydroclimatology of the Upper Madeira River basin: spatio-temporal variability and 69.Espinoza, J. C. et al. Regional hydro-climatic changes in the Southern Amazon Basin (Upper Madeira Basin) 70.Du, J., Fang, J., Xu, W. \& Shi, P. Analysis of dry/wet conditions using the standardized precipitation index an 71.Wang, Y., Chen, X., Chen, Y., Liu, M. \& Gao, L. Flood/drought event identification using an effective indicat 72.Seiler, R. A., Hayes, M. \& Bressan, L. Using the standardized precipitation index for flood risk monitoring. I 73.Frederick, I. O., Williams, M. A., Sales, A. E., Martin, D. P. \& Killien, M. Pre-pregnancy Body Mass Index, 74.McKee, T. B., Doesken, N. J. \& Kleist, J. The relationship of drought frequency and duration to time scales. 75.Umlauf, N., Klein, N. \& Zeileis, A. BAMLSS: Bayesian Additive Models for Location, Scale, and Shape (an 76.IBGE. Censo Demográfico 2010. Censo Demográfico 2010 http://ibge.gov.br/home/estatistica/populacao/cen 77.Blanc, A. K. \& Wardlaw, T. Monitoring low birth weight: an evaluation of international estimates and an upd 78...... Wood, S. N. Generalized additive models: an introduction with R. (Chapman and Hall, 2006). 
We assess how an individual newborn's prenatal exposure to climatic variability in their mother's municipality of residence may affect their birth-weight through growth or gestational age (GA); only growth; and only GA. We account for maternal social characteristics, prenatal and obstetric healthcare, and municipal characteristics. For each newborn, we draw on official birth certificates to obtain information on the timing and location of pregnancy, maternal characteristics, and healthcare received. Our analysis includes six key stages (E.D. Fig. 2).

\section{$\underline{\text { Step (1) Select study universe }}$}

We focus on live births in highly river-dependent municipalities in Amazonas State, Brazil, unconnected to other urban centres by road (n=43) (E.D. Fig. 1). These municipalities' populations have higher social vulnerability to climatic shocks than road-connected municipalities. High social vulnerability in these areas arises through adaptive capacity deficits, higher social sensitivity and higher prices of imported foodstuffs ${ }^{7}$. In these places, only very proximate rural communities are reachable by road/track from the urban centre. Most of them are only reachable by river. A global study found 'roadlessness' is associated with underdevelopment ${ }^{63}$.

\section{$\underline{\text { Step (2) Calculate and compare seasonal local rainfall and river-level anomalies }}$}

2a) Rainfall measurement. Precipitation was derived from the satellite-based Tropical Rainfall Measuring Mission (TRMM) project and the Global Precipitation Measurement (GPM) project, both NASA-JAXA collaborations. TRMM's mission ended in 2015 whereas GPM launched in 2014; we used NASA's combined rainfall data product, IMERG 'Final Run', Level 3. TRMM has been widely used in hydrological research, and IMERG has been validated for Brazil, including Amazonia $^{64,65}$. IMERG performs better than TRMM for smaller areas because of its finer spatial- 
temporal resolution ${ }^{66}$. IMERG provides rainfall measure every 3 hours for $0.25^{\circ} \times 0.25^{\circ}$ cells from 2002 to 2017 . We weekly-averaged measures by municipality, i.e. weekly-rainfall-per-municipality.

2b) River-level measurement. Historical river-levels were extracted for monitoring stations in Amazonas from 2004-to-2014, from the Hidroweb platform of Brazil's Agência Nacional de Águas. We could not extract comparable river-level data after 2014 due to changes in the platform. . Anomalies at municipality-level were obtained by extracting the residuals of harmonic regression models for each station active for $\geq 10$ years (S.M. Fig. 4). The residuals were interpolated onto a raster image using ordinary kriging, and then were averaged for each municipality and week.

2c) Comparing anomalies. We compared rainfall and river-level anomalies for 2004-to2014. After establishing the relatively strong (but spatially-differentiated) relationship between rainfall and subsequent river-level anomalies (E.D. Fig. 4) we chose satellite-derived intense/deficient rainfall episodes (section $4 b$ ) as our measure of climatic variability. Satellite-based precipitation is useful for understanding local climatic variability and even hydrological modelling in Amazonia, due to the low density of on-the-ground rainfall or river gauges ${ }^{67}$. In our study area some municipalities had long-term river-level gauges whereas others lacked local data, which would have required modelling local climatic variability using interpolation from stations hundreds of kilometers up/down-river, or in different sub-basins. Overall, we find local intense and deficient rainfall events are indicative of 'extreme climatic events' which often lead to riverine flooding or drought. Work from the Peruvian Amazon also shows a strong relationship between satellite-derived rainfall estimates and observed streamflow ${ }^{67}$. Evidence for using precipitation anomalies to capture river-level anomalies (and hence, river flooding and drought) is weaker in the main-stem. Even if local rainfall was normal, a main-stem municipality might experience a hydrological drought due to 
extremely low rainfall upriver, or a river flood due to extremely high rainfall upriver. Research also shows that river-levels in the Brazilian section of the Madeira River are strongly related to rainfall in the Peru and Bolivian Amazon $3,45,68,69$.

\section{Step (3) Gestational timing and location}

Data were extracted from the Sistema de Informação sobre Nascidos Vivos (SINASC), Brazil's health information system for registering live births. During the study period (01/2006-to-12/2017) there were 291,479 live births recorded in our study region, where mothers had pregnancy duration $\geq 22$ weeks, for singleton births without congenital abnormalities. Records are based on official birth certificates with information on newborn's weight, birth circumstances and mother's sociodemographic position (section 6c). Maternal municipality of residence was used to specify newborn's municipality, and hence their prenatal rainfall variability exposure, and additional municipality characteristics (section 6b). The difference between birth-date and approximate GA was used to estimate conception date and the exposure period to rainfall variability. Because GA was recorded in categorical intervals (e.g. [22-27] weeks), the middle-point was used as the approximate GA (eg. $24.5=(22+27) / 2)$.

\section{Step (4) Calculate prenatal exposure to three magnitudes of rainfall variability}

For each newborn, we calculated prenatal exposure (including pre-pregnancy trimester) to extreme and non-extreme rainfall events, and seasonal deviations using the timing, duration and location of pregnancy, and an improved model-based version of the Standardized Precipitation Index (SPI). We utilize a Model Based Standardised Index (MBSI), used for measuring the magnitude of intense/deficient rainfall events. It models seasonal rainfall distribution from historical data, computes the relative position of the observations in terms of probabilities, and transforms them to normal standard quantiles. For details, including advantages relative to the SPI, $\operatorname{see}^{44}$. The MBSI 
provides a measure of deviation from seasonal behaviour with respect to the moving average rainfall of order $k$, called the 'time-scale'. We assessed rainfall variability at two time-scales; 1week (for deviations from seasonal averages) and 8-weeks (to identify extreme and non-extreme events based on cumulative anomalies during this time period). We computed MBSI at an 8-week time-scale $(k=8)$ because; (1) this time-scale been used to identify major river floods and droughts in China ${ }^{70}$ with the maximum correlation between river discharge and $\mathrm{SPI}^{71}$, and was reliable for flood-risk monitoring in Argentina ${ }^{72}$; (2) Our analysis of SPI time-scale in Amazonas shows 8weeks is reliable for identifying extreme climatic events ${ }^{44}$.

We developed a bivariate index to capture deviation from average seasonal rainfall, and formulated a separate measure of joint exposure to intense and deficient rainfall events (or extremes of each) during (pre-)pregnancy. The pre-pregnancy trimester (12 weeks) was taken as immediately prior to the estimated conception date. These indices accounted for the duration of each pregnancy in order to avoid confounding results because GA influences birth-weight. Maternal nutritional status pre-pregnancy strongly influences birth outcomes ${ }^{73}$. We therefore include measures of prepregnancy climatic variability because exposure might affect maternal health or nutrition prior to conception, potentially influencing fetal development ${ }^{11,50}$. Moreover, climatic shocks might have delayed impacts on local food access ${ }^{21}$ hence a pre-pregnancy shock may disrupt maternal food intake and nutrition several months later.

4a. Precipitation deviations. We assessed deviation from seasonal behaviour of rainfall using a one-week time-scale $(k=1)$ when computing MBSI. We represent the obtained MBSI for mother of newborn $i$ at week of pregnancy $j$ as $S_{i j}^{k=1}$, where $i=1, \ldots, m$ and $j=-11, \ldots, 0, \ldots, d_{i}$. Possible values of $j$ are from 12 weeks before the mother was pregnant until the pregnancy duration $d_{i}$. The sum of only positive and only negative deviations during the pre-pregnancy and pregnancy period divided by the number of weeks are the elements our bivariate indicator $D_{i}$ : 
$D_{i}=\left(D_{i 1}, D_{i 2}\right)=\left(\sum_{j=-11}^{d_{i}} \frac{s_{i j}^{k=1} \delta_{\left(s_{i j}^{k=1}<0\right)}}{d_{i}+12}, \sum_{j=-11}^{d_{i}} \frac{s_{i j}^{k=1} \delta_{\left(s_{i j}^{k=1}>0\right)}}{d_{i}+12}\right)$,

where $\delta(c)$ takes a value 1 when the underlying condition, $c$, holds and 0 otherwise. $D_{i 1}$ and $\mathrm{D}_{i 2}$ measures negative and positive deviations respectively. Low values in both dimensions represent a mother's exposure close to historical seasonal rainfall. Cases where $\mathrm{D}_{\mathrm{i} 2}$ is high and $D_{i 1}$ close to zero, represent mothers experiencing higher rainfall than expected. Conversely, high values of $D_{i l}$, and $D_{i 2}$ close to zero represent mothers experiencing less rainfall than expected.

4b. Intense and deficient rainfall events. A deficient (or intense) rainfall event is a period when the MBSI is continuously negative (or positive, for intense rainfall) reaching at least one value lower (or higher for intense events) or equal to -1 ( 1 for intense events) $)^{74}$. The sum of standardized precipitation values corresponding to deficient (or intense) rainfall events during prepregnancy and pregnancy divided by the number of weeks are the elements of our bivariate indicator $D F_{i}$ for measuring exposure to intense and deficient events such as:

$$
D F_{i}=\left(\sum_{j=-11}^{d_{i}} \frac{S_{i j}^{k=8} \delta_{(j \in \text { deficientrainfallevent })}}{d_{i}+12}, \sum_{j=-11}^{d_{i}} \frac{S_{i j}^{k=8} \delta_{(j \in \text { intenserainfallevent })}}{d_{i}+12}\right)
$$

4c. Extreme rainfall events. We computed exposure to extreme events $E_{i}$ similarly to exposure to $D F_{i}$, but only considered the 8 -week MBSI values $>2$ or $<-2$. These limits are conventional for characterizing extreme intense and deficient rainfall events, respectively ${ }^{74}$. Our bivariate index for mother of newborn $i$ is defined as:

$E_{i}=\left(\sum_{j=-11}^{d_{i}} \frac{s_{i j}^{k=8} \delta_{\left(s_{i j}^{k=8}<-2\right)}}{d_{i}+12}, \sum_{j=-11}^{d_{i}} \frac{s_{i j}^{k=8} \delta_{\left(s_{i j}^{k=8}>2\right)}}{d_{i}+12}\right)$

\section{$\underline{\text { Step (5) Define local hydrological seasons }}$}


Using river-level data from 2004-2014, we defined hydrological seasons by municipality. To measure and standardise seasonality of river-levels, we fitted harmonic regression models from stations active for $\geq 10$ years. The harmonic terms were chosen using forward selection. For each station, we identified the annual period when rivers, on average, reached their highest levels. We transformed these series into seasonal status (values between 0 and 53): weeks 0 and 53 (cyclically) denote peak wetness, whereas values around 26 are peak dryness. We interpolated them spatially onto a raster image using ordinary kriging. Lastly, we weekly-averaged the resulting pixel-level data by municipality; the seasonal river-level index. Amazonas State is enormous therefore the calendar week of peak wetness varies greatly, particularly between the north and south (E.D. Fig. 4). For each newborn, we controlled for hydrological seasonality by using the hydrological-week in their mother's municipality of residence at estimated date of conception.

\section{Step (6) Bayesian modelling of mean birth-weight, low birth-weight and preterm birth}

\section{6a) Hierarchical modelling approach.}

We modelled how rainfall variability affects birth-weight through intra-uterine growth or GA; only growth; and only GA using MBSI, together with municipality and maternal characteristics, and temporal and municipality effects. Our individual (newborn)-level analysis is nested at municipality-level. We model birth-weight (M.1) and LBW (M.3) without controlling for GA to assess the full effects; birth-weight (M.2) and LBW (M.4) controlling for GA to assess the effect through growth; and PTB (M.5) to assess to effect through GA. We model the location, scale and probability parameters using:

Exposure to rainfall variability (intense/deficient) of 3 magnitudes (extreme, non-extreme events, seasonal deviations)

- Seasonal effects (river-levels)

- Municipality-level covariates 
- Individual-level covariates (newborn, mother, healthcare)

- Unexplained temporal effects (using conception's week)

- Unexplained municipality effects

We used Bayesian additive models for location, scale and shape (BAMLSS) linking the parameters with linear predictors that depend on covariates; non-linear functions of covariates; and independent random effects. Bayesian inference is achieved by obtaining the mode of the posterior distribution and later using these as starting values to obtain samples of the posterior distribution through Monte 590 Carlo Markov chain algorithms; see $^{75}$ for detailed explanation. This model is an extension of a generalized linear model (GLM) in which, in addition to the location parameter, the scale and shape parameters are also modelled through linear predictors that allow the inclusion of smooth functions to capture different effects types such as univariate non-linear, spatial and temporal effects and nonlinear interactions ${ }^{75}$. We used BAMLSS because; (I) Exploratory analysis revealed heteroscedasticity across certain covariates levels (e.g. maternal age), indicating the need to also model the scale parameter. (II) non-linear effects using basis functions were less computationally expensive compared to other alternatives (e.g. Gaussian processes). (III) BAMLSS models allow including non-linear interactions to evaluate the effects of our bivariate indices that jointly measure maternal exposure to intense and deficient rainfall events. A more simplistic approach with separate measures of exposure to intense/deficient rainfall events would lead to misleading results.

\section{6b) Municipal characteristics}

We used 2010 Brazilian census data to define the proportion of households with an internal tap and toilet for each municipality ${ }^{76}$. Remoteness was measured using an index of travel distances from each urban centre to from larger towns/cities in a hierarchical urban network with values from 0 (least remote) to 1 (most remote) $\left(\mathrm{see}^{7}\right)$. 


\section{6c) Birth-weight and maternal characteristics}

SINASC data also provided: birth-weight (grams); categorized gestational age (22-27 weeks; 28$31 ; 32-36 ;>37$ ), sex of infant (male/female); ethnicity of newborn as reported by the mother (reclassified as indigenous Amerindian or not); maternal age (years), marital status (single/married/civil union/widowed/divorced) and formal education received (0 years; $1-3 ; 4-7 ; 8$ $11 ; \geq 12)$; antenatal consultations $(0 ; 1-3 ; 4-6 ; \geq 7)$; birth-place (hospital/health center/ home/other location). There were missing values for birth-weight (2.1\%), marital status (22\%), type of birthplace $(0.006 \%)$, ethnicity $(0.4 \%)$, antenatal consultations $(1.1 \%)$ and mother's age $(0.001 \%)$. One registration missing birth-weight and mother's age was removed. To handle more prevalent missing data for other covariates we introduced "missing" levels. Our dataset did not include unique identifiers for mothers therefore, unavoidably, some mothers will appear several times in our database if they gave birth $\geq 2$ during the study period. certain values, such as $13 \%$ of records being multiples of $500 \mathrm{~g}$. This problem, 'heaping', can occur if a birth was recorded without due diligence or if the mother reports an approximate value because the newborn was not weighed at-birth. The latter is more likely in rural areas where mothers tend to have lower education and less antenatal care hence care must be taken when interpreting analyses based on these data ${ }^{77}$. This problem could lead to differences in the observed birth-weight variability across municipalities; we take this into account by modelling the standard deviation with respect to municipality.

6d) Statistical modelling. Five types of BAMLSS models were used: birth-weight (M.1) and LBW (M.3) without controlling for GA; birth-weight (M.2) and LBW (M.4) controlling for GA; and PTB (M.5). We evaluated distribution adequacy when modelling birth-weight (M.1 and 
M.2); importance of including rainfall variability for each model type; and sensitivity to hyperparemeters for model M.3 and M.5 only because of the computational cost for other models (e.g. birth-weight). The non-linear effects in our models are represented using thin plate regression splines; a low rank approximation of thin plate splines obtained by minimizing a cost function that considers the trade-off between model fitting and smoothness, and does not require knots (see ${ }^{78}$ ).

\section{6d.i) Proposed models}

Gaussian and t-student distributions with mean $(\mu)$ and scale $(\sigma)$ parameters were used to model birth-weight, while the Bernoulli distribution with probability parameter $(\pi)$ was used for LBW and PTB. The linear predictor for the scale parameter for birth-weight models (M.1 and M.2) was defined as:

$$
\begin{gathered}
\log \left(\sigma_{i j}\right)=\eta_{i j}^{*}=\beta_{0}^{*}+h_{1}^{*}\left(\text { sex }_{i j}\right)+h_{2}^{*}\left(\text { yearsofeducation }_{i j}\right)+h_{3}^{*}\left(\text { birthplace }_{i j}\right)+h_{4}^{*}\left(\text { ethnicrace }_{i j}\right)+ \\
h_{5}^{*}\left(\text { consultationsnumber }_{i j}\right)+f_{1}^{*}\left(\text { age }_{i j}\right)+f_{2}^{*}\left(\text { conceptiondat }_{i j}\right)+r^{*}\left(\text { municipality }_{j}\right)
\end{gathered}
$$

where $\sigma_{\mathrm{ij}}$ is the standard deviation for newborn $i$ in municipality $j$ and $\beta_{0}^{*}$ is the intercept. The functions $h^{*}{ }_{1}(),. h_{2}^{*}(),. \ldots, h^{*}$ (.) represent the effects of categorical variables, $f^{*}{ }_{1}(),. f^{*}{ }_{2}($. represent univariate thin plate regression splines, and $r^{*}($.$) represents independent random effects.$

The linear predictors associated with the mean $\left(\mu_{i j}=\eta_{i j}\right)$ or probability parameters $\left(\operatorname{logit}\left(\pi_{i j}\right)=\eta_{i j}\right)$, for child $i$ in municipality $j$, for models without controlling for GA (M.1, M.3 and M.5) were defined

as:

$$
\begin{gathered}
\eta_{i j}=\beta_{0}+h_{1}\left(\text { sex }_{i j}\right)+h_{2}\left(\text { maritalstatus }_{i j}\right)+h_{3}\left(\text { yearsofeducation }_{i j}\right)+h_{4}\left(\text { birthplace }_{i j}\right)+h_{5}(\text { ethnicr } \\
\left.h_{6}\left(\text { consultationsnumber }_{i j}\right)+f_{1}\left(\text { age }_{i j}\right)+f_{2}\left(\text { conceptiondate }_{i j}\right)+\text { s }_{\text {riverlevelweek }}\right)+ \\
l_{1}\left(\text { remoteness }_{j}\right)+l_{2}\left(\text { taptoiletproportion }_{j}\right)+r\left(\text { municipality }_{j}\right)+ \\
f_{3}\left(D_{i 1}, D_{i 2}\right)+f_{4}\left(D F_{i 1}, D F_{i 2}\right)+f_{5}\left(E_{i 1}, E_{i 2}\right),
\end{gathered}
$$

Functions $h_{1}(),. \ldots, h_{6}(.) ; l_{1}(),. l_{2}($.$) represent fixed effects of factor and continuous variables$ respectively. Functions $f_{1}(),. f_{2}($.$) represent univariate thin plate regression splines, while f_{3}(),. f_{4}($.$) ,$ 
$f_{5}($.$) are bivariate. Lastly, s($.$) and \mathrm{r}($.$) represents cyclic and cubic regression splines used for$ modelling seasonality and indepent random effects respectively. Previous analysis led us to include linear effects for remoteness and sanitation to avoid over-fitting; exclude rurality (rural \% of municipal population) because it was correlated with sanitation $(\mathrm{r}=-0.33)$ and was not significant (at 95\%); and include independent random effects at municipality-level to account for unexplained municipality differences and intra-municipality correlation.

The linear predictors of models controlling for GA (M.2 and M.4) include the term $h_{7}$ (gestational $\left.a g e_{i j}\right)$; and the alternative models without including rainfall variability remove the terms $f_{3}\left(\mathrm{D}_{\mathrm{i} 1}, \mathrm{D}_{\mathrm{i} 2}\right)$, $f_{4}\left(\mathrm{Df}_{\mathrm{i} 1}, \mathrm{DF}_{\mathrm{i} 2}\right), f_{5}\left(\mathrm{E}_{\mathrm{i} 1}, \mathrm{E}_{\mathrm{i} 2}\right)$ from the linear predictors.

665 The priors depend on the semi-parametric representation of the effects $h_{i}(),. f_{i}(),. s_{i}(),. h_{i}{ }^{*}($.$) , and$ $f_{i}^{*}($.$) , for which the BAMLSS package prefers to define weakly informative priors \left({ }^{75}\right.$, Section 4.1$)$.

\section{5d.ii) Model selection}

670 When modelling birth-weight (M.1), the t-distribution (DIC: 4420308) performed better than the Gaussian distribution (DIC: 4433581) in terms of goodness-of-fit and model assumptions' adequacy (comparing QQ-plots) due to the heavy tails of the residuals. For all models (M.1-M.5) we also found that fitting was improved by including rainfall variability (DIC: 4420308, 129549, 4408755, 120447.2, 152947.9) compared to not including (DIC: 4420543, 129784.3, 4408848, 120464.6, 160983). This shows the relevance rainfall variability on birth outcomes. There was no strong evidence to support the inclusion of spatial effects at with exception of model M.5 (P-value $=0.04)$; tested using the Moran index and Monte Carlo simulation. However, we did not include this effect because of convergence problems when including both spatial and independent municipality effects. 
The bivariate non-linear terms of our models might be difficult to interpret (E.D. Fig. 7). To facilitate interpretation, we computed credible intervals for three (or four) bivariate combinations representing different scenarios like high exposure to extreme intense (or deficient) episodes, moderate exposure to extreme intense and deficient rainfall (Fig. 4).

\section{6d.iii) Bayesian inference}

The number of chains, iterations, burn-in and thinning for each model was defined by considering the RAM required to run parallel chains; and inspecting convergence using traceplots, autocorrelation function plots. These graphs can be seen at https://erickchacon.gitlab.io/birthweight/. For model M.1, we ran 3 chains of 7000 iterations with no burn-in and keeping 1/20 samples. For M.3, we ran 3 chains of 9000 iterations removing the first 1000 iterations and keeping 1/20 samples. For M.2 and M.4, we ran 4 chains of 7000 iterations removing the first 3500 iterations and keeping 1/10 samples. For M.5, we ran 4 chains of 10000 iterations removing the first 3500 iterations and keeping 1/20 samples. Consistently, for most models, convergence was slower for the independent random effects, municipality covariates 695 (remoteness and sanitation), and the intercept.

We assessed sensitivity to the priors's hyperparameters of the inverse smoothing parameters for models M.3 and M.5 with three types of priors using an inverse gamma distribution with shape $a$ and rate $b$ : (i) weakly informative prior (default; $\mathrm{a}=b=0.0001$ ), (ii) informative prior in favor of non-smooth functions $(a=5, b=1000)$, and (iii) informative prior in favor of smooth functions ( $\mathrm{a}=$ $10, \mathrm{~b}=0.0001$ ). Highly informative priors (ii and iii) influenced the estimated terms and their uncertainties, but similar patterns on the effects were observed. In absence of strong knowledge about the inverse smoothing paramaters, we base our results on weakly informative priors (i). 
705 Acknowledgements This work was supported by grants from the UK (ESRC ES/K010018/1, Newton ES/M011542/1), Brazil (CNPq PVE 313742/2013-8) and the European Commission (H2020 RISE 691053 ODYSSEA). Useful discussion and support was provided by Fiocruz colleagues A. Cardoso, M. Theme, A.A da Silva and comments from N. Graham and J. Barlow. We are grateful to five reviewers for their very helpful suggestions.

710 Author contributions E.C.M., L.P., B.M.T. and M.G.C. designed the research with additional input from J.D.Y.O. G.D. developed the network analysis for spatial remoteness measures. E.C.M. analyzed the data with input from B.M.T, M.G.C. and L.P. J.D.Y.O. supported data interpretation. L.P. and E.C.M. wrote the manuscript, with input from all authors.

Author information. Reprints and permissions information is available at

715 www.nature.com/reprints. The authors declare no competing interests. Correspondence and requests for data and coding should be addressed to L.P. (luke.parry@lancaster.ac.uk). 